\title{
FINITE THREE-DIMENSIONAL SURFACE WAVES IN CIRCULAR CHANNELS*
}

\author{
BY \\ LESTER Q. SPIELVOGEL
}

National Oceanic and Atmospheric Administration Joint Tsunami Research Effort, Honolulu

\begin{abstract}
This paper is concerned with finite surface waves in incompressible fluid in circular channels or bowls. Fluid circulates through these channels and is contained in them by the action of an axial gravitational force. Under certain velocity conditions permanent waves of finite magnitude may be solutions to the differential equations of motion. These waves are related to the cnoidal and solitary waves of the infinite straight channel. Using stretching techniques on the nonlinear differential equations, we derive expressions for the wave shape, the critical velocity condition, and relations to waves in straight channels. The irrotational case is thoroughly treated and a short discussion of the rotational case is included. The fact that these waves deform continuously into those already derived for straight channels is also discussed. The most important result, however, is that stretching techniques have been shown to describe finite waves progressing in curved trajectories. While it is true that the trajectories are dictated by the curvature of the containing vessels, it is apparent that this technique may very well be useful in describing finite wave shape and trajectories for waves near beaches and breakwaters.
\end{abstract}

Introduction. Since 1944, when Scott Russell made his first observations of a solitary wave in a canal, a considerable amount of theory has been developed dealing with solitary waves in fluids. Most of this theory is concerned with two-dimensional flow and flow in infinite straight channels. In this paper we shall be concerned with threedimensional cnoidal waves in circular channels and circular bowls. We will find that the solutions of this problem have several points in common with previous results. The first of these is the characteristic critical speed. For certain velocity profiles the equations will exhibit a bifurcation, i.e. there will be two families of solutions. The first family, which will be called the steady-flow state, will exhibit no wave in the direction of flow. This family of solutions is also common to flows at noncritical speeds. The second family of solutions will be nontrivial waves of a permanent type; i.e., the basic shape of the wave is fixed although it moves over the surface of the fluid. The second point in common with previous works in this subject will be the characteristic cnoidal profile (Jacobi elliptic function) in the direction of flow. A third point in common with previous work is that the specific wave shape depends on the solution of an elliptic Neumann problem in the steady-flow cross-section of the fluid.

This paper will be devoted to an examination of irrotational inviscid progressing waves of permanent shape. These steadily progressing waves are waves whose shape remains fixed as viewed from a coordinate system which is rotating with respect to the

\footnotetext{
* Received November 20, 1970.
} 
fixed coordinate system at a constant angular velocity. When the angular velocity is zero, these waves will be called stationary waves.

We will be interested in waves in circular channels or bowls. It will be obvious from the solutions that the two will be equivalent except for some pathological bowls with certain infinite dimensions. Consider a fixed curve $s-G(\rho)=0$ in a cylindrical coordinate system where $\rho$ is the radial coordinate, $\alpha$ is the angular coordinate, and $s$ is the axial coordinate (see Fig. 1). This fixed curve is rotated about the axis $\rho=0$ of the coordinate system, and thus it generates a channel or bowl. Fluid will be constrained in this channel by the action of a constant gravitational force along this same axis.

It is not a priori clear that cnoidal/solitary waves exist in circular channels since their existence in straight infinite channels is strongly coupled with the fact that waves of very large wavelength exist in such channels. The infinite length of the straight channels, in which it has been proven that cnoidal waves do exist, stands in contrast to the finite length of the liquid-filled circular channel discussed herein. The existence of these waves in finite channels is suggested by observations. In particular, the solitary waves observed for finite times under natural circumstances and also under laboratory conditions in finite channels strongly suggest that these permanent waves do exist. It is for these reasons that we apply variations of the techniques for infinite straight channels to the problem of circular channels. What we do, basically, is associate a stretching parameter to some of the dependent and independent variables so as to emphasize the angular direction and angular velocity. We then expand each of the new dependent variables in a power series in this parameter. The basic assumption is that these power series converge to solutions or represent solutions in an asymptotic sense. We substitute these series into the equations coefficient to different powers of the stretching parameter. That is, the equation $L(x, \epsilon, \phi(x, \epsilon))=0$, where $L$ is a differential operator (or boundary condition), $x$ represents time and space variables, $\epsilon$ is the stretching parameter, and $\phi$ is the desired solution, will yield the following expression under the assumption $\phi(x, \epsilon)=\sum_{i=0}^{\infty} \phi_{i}(x) \epsilon^{i}$ :

$$
L=\sum_{i=0}^{\infty} L_{i}\left(x, \phi_{0}, \cdots, \phi_{i}\right) \epsilon^{i}=0 .
$$

In this expression the $L_{i}$ are differential operators (or boundary conditions) independent of $\epsilon$. These resulting equations are solved recursively starting with those coefficient to the lowest powers of the stretching parameter. This assures us of consistent results from all the equations. The results in all the cases considered show that the leading terms in the expansion of the wave profile have a marked cnoidal shape in the angular direction. The shape of the wave in the radial direction is highly dependent on the steady-flow fluid velocity and the velocity of the progressing wave.

Equations of motion. Let the spatial coordinates by $(\rho, \alpha, s)$ and let them be indexed 1, 2, 3 as in Fig. 1. The velocity vector $\mathrm{V}$ in this cylindrical coordinate system is

$$
\mathrm{V}=\mathrm{i}_{1} V_{1}+\mathrm{i}_{2} V_{2}+\mathrm{i}_{3} V_{3},
$$

where $V_{1}=d \rho / d t, V_{2}=\rho(d \alpha / d t)$, and $V_{3}=d s / d t$. The velocity $\mathbf{V}$ will be referred to in the body of this report as the fluid velocity and as the particle velocity.

The equations of the free surface and the fixed surface will be, respectively:

$$
s=\eta(\rho, \alpha, t)
$$



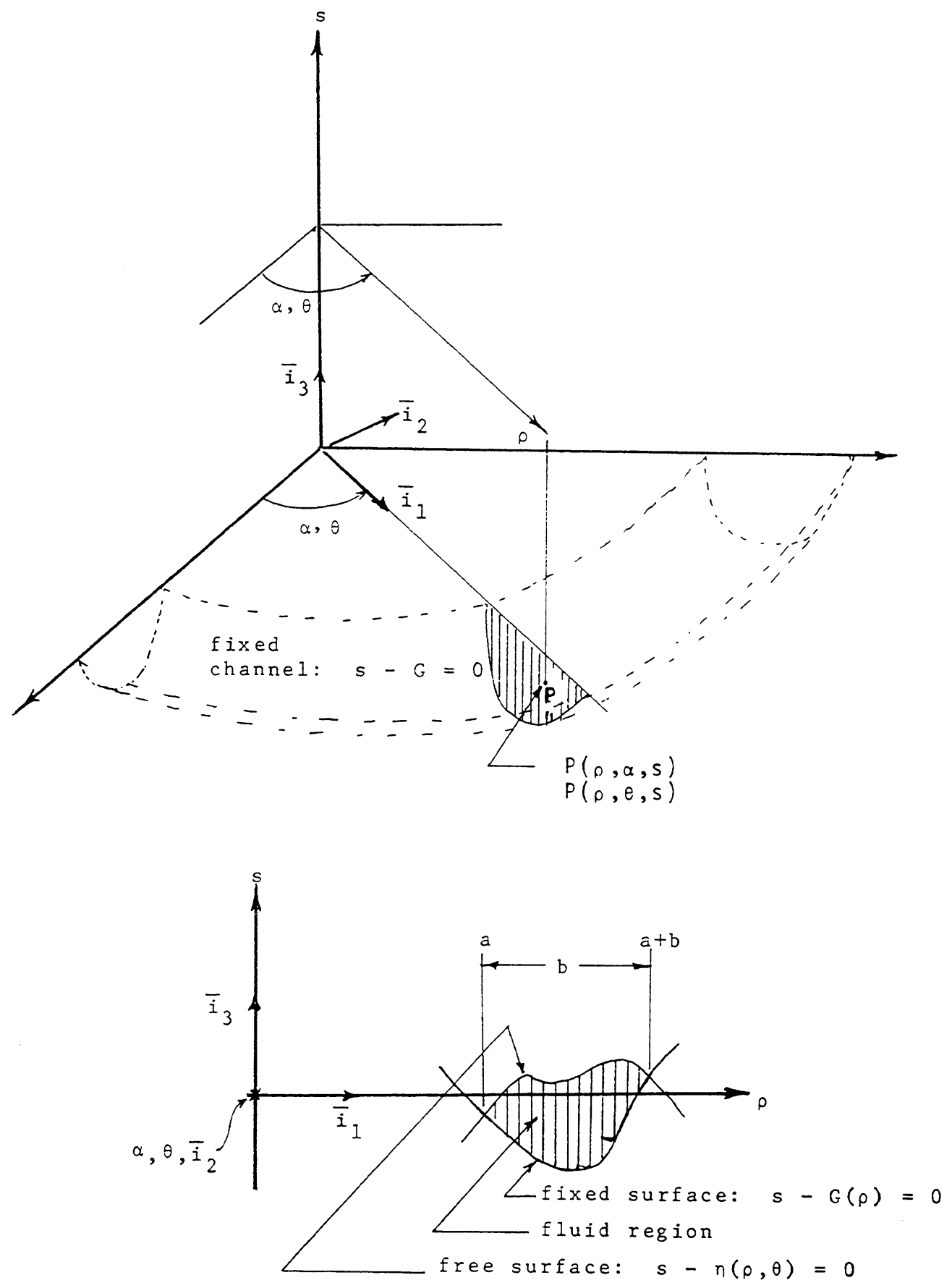

Fig. 1. Coordinate system. 
and

$$
s=G(\rho) .
$$

The equations of continuity and momentum are given respectively by

$$
\nabla \cdot V=0
$$

and

$$
\mathbf{F}-(1 / \delta) \operatorname{grad} P=d \mathbf{V} / d t
$$

In these equations $P$ is the pressure, $t$ is time, $\delta$ is the constant density, and $\mathbf{F}$ is the constant body force. To be specific, we will solve the problem with

$$
\mathbf{F}=-\mathbf{i}_{3} g
$$

where $g$ is a positive constant. We can think of the fluid as held in a circular channel (3) by the action of a gravity force (6) directed along the axis of symmetry $\rho=0$.

It is best to solve these equations in the Eulerian form. The solution will then be given by the velocity in terms of the space and time coordinates. In the fluid interior we have

$$
\begin{gathered}
\frac{1}{\rho} \frac{\partial}{\partial \rho}\left(\rho V_{1}\right)+\frac{1}{\rho} \frac{\partial}{\partial \alpha} V_{2}+\frac{\partial}{\partial s} V_{3}=0, \\
V_{1 \iota}+V_{1} V_{1 \rho}+\frac{1}{\rho} V_{2} V_{1 \alpha}+V_{3} V_{1,}-\frac{1}{\rho}\left(V_{2}\right)^{2}=-\frac{1}{\delta} P_{\rho}, \\
V_{2 \imath}+V_{1} V_{2 \rho}+\frac{1}{\rho} V_{2} V_{2 \alpha}+V_{3} V_{2 s}+\frac{1}{\rho}\left(V_{1} V_{2}\right)=-\frac{1}{\delta \rho} P_{\alpha}, \\
V_{3 \imath}+V_{1} V_{3 \rho}+\frac{1}{\rho} V_{2} V_{3 \alpha}+V_{3} V_{3,}=-\frac{1}{\delta} P_{\imath}-g .
\end{gathered}
$$

These partial differential equations will be solved subject to zero pressure on the free surface and the natural kinematic boundary conditions. On $s=\eta(\rho, \alpha, t)$,

$$
P=0
$$

and

$$
V_{3}=\eta_{\rho} V_{1}+\frac{1}{\rho} \eta_{\alpha} V_{2}+\eta_{t}
$$

and on $s=G(\rho)$,

$$
V_{3}=\frac{d G(\rho)}{d \rho} V_{1}
$$

We now introduce a stretching technique to facilitate finding a solution to the problem. Let $\epsilon$ be the dimensionless stretching parameter. Then coordinates $\rho, \alpha, s$ go into $\rho, \theta, s$ and $P, V_{1}, V_{2}, V_{3}, t$ go into $\pi, U, V, W, \tau$ respectively:

$$
\begin{gathered}
\theta=\sqrt{\epsilon} \alpha, \quad \tau=\sqrt{\epsilon g} t, \quad U=V_{1} \sqrt{\epsilon / g}, \\
V=V_{2} \sqrt{1 / g}, \quad W=V_{3} \sqrt{\epsilon / g}, \quad \pi=P /(\delta g) .
\end{gathered}
$$


We solve the problem in the new coordinate system and then use Eq. (14) to identify the old variables. Eqs. (7) through (13) become Eqs. (15) through (21). In the fluid region we solve

$$
\begin{aligned}
& \epsilon \frac{1}{\rho}\left(V_{\theta}\right)+\frac{1}{\rho}(\rho U)_{\rho}+W_{\iota}=0, \\
& \epsilon\left(U_{\tau}+\pi_{\rho}+\frac{1}{\rho} V U_{\theta}-\frac{1}{\rho} V^{2}\right)+U U_{\rho}+W U_{\iota}=0, \\
& \epsilon\left(V_{\tau}+\frac{1}{\rho} \pi_{\theta}+\frac{1}{\rho} V V_{\theta}\right)+\frac{1}{\rho} U V+U V_{\rho}+W V_{\iota}=0, \\
& \epsilon\left(W_{\tau}+\pi_{\odot}+\frac{1}{\rho} V W_{\theta}+1\right)+U W_{\rho}+W W_{\iota}=0 .
\end{aligned}
$$

On the free surface $s-\eta(\rho, \theta, \tau)=0$ we have

$$
\pi=0
$$

and

$$
W-U \eta_{\rho}=\epsilon\left(\eta_{\tau}+V \eta_{\theta} / \rho\right) .
$$

On the fixed surface $s-G(\rho)=0$ we have

$$
W-U G^{\prime}(\rho)=0 .
$$

(Note: in what follows we shall use the following alternate conventions: $\partial() / \partial \rho=\left(^{\prime}\right)$, $\partial() / \partial \theta=()$.) For irrotational waves we set the vorticity equal to zero:

$$
\nabla \times \mathrm{V}=\mathbf{0} .
$$

This implies the existence of a potential function $\phi$ such that

$$
U=\phi_{\rho}, \quad V=\frac{1}{\rho} \phi_{\theta}, \quad W=\phi_{\mathbf{s}} .
$$

It is now evident that we can integrate Eqs. (16), (17) and (18) to get an energy integral. The problem is then reduced to finding a solution of the following equations.

In the interior of the fluid we solve

$$
\frac{\epsilon}{\rho^{2}} \phi_{\theta \theta}+\frac{1}{\rho} \frac{\partial}{\partial \rho} \rho \frac{\partial}{\partial \rho} \phi+\phi_{s}=0 .
$$

On the free surface $s=\eta(\rho, \theta, \tau)$ we have the boundary conditions

$$
\epsilon\left(\eta_{\tau}+\frac{1}{\rho^{2}} \phi_{\theta} \eta_{\theta}\right)=\phi_{s}-\phi_{\rho} \eta_{\rho}
$$

and

$$
\left(E(\tau)-\frac{1}{2}\left(\phi_{\theta}\right)^{2}-\frac{1}{2}\left(\phi_{\rho}\right)^{2}\right)=\epsilon\left(\eta+\phi_{\tau}+\frac{1}{2 \rho^{2}}\left(\phi_{\theta}\right)^{2}\right) .
$$

On the fixed surface $s=G(\rho)$ we have

$$
\phi_{s}-G^{\prime}(\rho) \phi_{\rho}=0 .
$$


We may then solve for the pressure by using Eq. (28):

$$
\pi=\frac{1}{\epsilon}\left[E(\tau)-\frac{1}{2}\left(\phi_{\rho}\right)^{2}-\frac{1}{2}\left(\phi_{s}\right)^{2}\right]-\left[\phi_{\tau}+s+\frac{\left(\phi_{\theta}\right)^{2}}{2 \rho^{2}}\right] .
$$

Irrotational progressing waves. We now proceed to investigate progressing waves. We have already set up the equations for incompressible, irrotational, inviscid fluid flow in a circular channel with both a free and a fixed boundary. The progressing waves that we defined earlier are characterized by the dependent variables being functions of ( $\rho . s, \alpha-\tilde{\Omega} t)$ in the original coordinates and $(\rho, s, \theta-\tilde{\omega} \tau)$ in the stretched coordinates. The two constants $\tilde{\Omega}$ and $\tilde{\omega}$ are fixed with respect to each other by

$$
\tilde{\omega}=\tilde{\Omega} / \sqrt{ } g .
$$

It follows that Eqs. (24) through (28) reduce to Eqs. (30) through (34). We note also that $E(\tau)$ reduces to a constant. We will solve Eqs. (30) through (34) as functions of $\rho, s, \theta$ with the understanding that we will substitute $\theta-\tilde{\omega} t$ for $\theta$ after the solutions are found.

In the fluid region we have

$$
\left(\frac{\epsilon}{\rho^{2}} \frac{\partial^{2}}{\partial \theta^{2}}+\frac{1}{\rho} \frac{\partial}{\partial \rho} \rho \frac{\partial}{\partial \rho}+\frac{\partial^{2}}{\partial s^{2}}\right)_{\phi}=0 .
$$

On $s-\eta(\rho, \theta)=0$

$$
\phi_{s}-\phi_{\rho} \eta_{\rho}=\epsilon \eta_{\theta}\left(\frac{\phi_{\theta}}{\rho}-\tilde{\omega}\right)
$$

and

$$
\eta=\frac{1}{\epsilon}\left(E-\frac{1}{2}\left(\left(\phi_{s}\right)^{2}+\left(\phi_{\rho}\right)^{2}\right)\right)-\phi_{\theta}\left(\frac{\phi_{\theta}}{2 \rho^{2}}-\tilde{\omega}\right) .
$$

On $s-G(\rho)=0$

$$
\phi_{.}-\phi_{\rho} G^{\prime}(\rho)=0 .
$$

After the above are solved for $\phi, \eta$, and $\tilde{\omega}$, the pressure may be determined directly from

$$
\pi(\rho, \alpha, s)=\frac{1}{\epsilon}\left(E-\frac{1}{2}\left(\left(\phi_{\rho}\right)^{2}+\left(\phi_{s}\right)^{2}\right)\right)-s-\phi_{\theta}\left(\frac{\phi_{\theta}}{2 \rho^{2}}-\tilde{\omega}\right) .
$$

We now substitute the formal power series (or asymptotic series) solution (35), (36) and (37) into Eqs. (30) through (34):

$$
\begin{aligned}
\phi(\rho, \theta, s ; \epsilon) & =\sum_{i=0}^{\infty} \phi_{i}(\rho, \theta, s) \epsilon^{i}, \\
\eta(\rho, \theta ; \epsilon) & =\sum_{i=1}^{\infty} \eta_{i}(\rho, \theta) \epsilon^{i} \\
E & =\sum_{i=0}^{\infty} E_{i} \epsilon^{i}
\end{aligned}
$$


We complete the expansion of (31) and (32) in the manner described in the introduction; as a result, Eqs. (30) through (34) are in the generalized form

$$
L=\sum_{i=0}^{\infty} L_{i}\left(x, \phi_{0}, \cdots, \phi_{i}\right) \epsilon^{i}=0
$$

where the $\left(L_{i}\right)$ are differential equations or boundary conditions. We note also that these expansions simplify the boundary conditions given by Eqs. (31) and (32). Instead of being evaluated on $s=\eta(\rho, \theta ; \epsilon)$ all the terms are evaluated on $s=\eta(\rho, \theta ; 0)$ or, more simply, on $s=\eta_{0}(\rho, \theta)$. Therefore, once we have determined $\eta_{0}(\rho, \theta)$ the problem reduces to that of a fixed boundary problem. We will see that the only admissible functions $\eta_{0}(\rho, \theta)$ will be exact steady-flow solutions to the partial differential equations. Hence, we can speak of this method as a finite-amplitude perturbation from the steadyflow solution.

The equations coefficient to $\epsilon^{0}$ are examined first. In the region $R$ we have

$$
\left(\frac{\partial}{\partial \rho} \rho \frac{\partial}{\partial \rho}+\frac{\partial}{\partial s} \rho \frac{\partial}{\partial s}\right) \phi_{0}=0 .
$$

On the boundary ( $\partial \mathrm{R}$ ) which is composed of segments of $s-\eta_{0}=0$ and $s-G(\rho)=0$ we have

$$
\frac{\partial}{\partial n}\left(\phi_{0}\right)=0
$$

On $s-\eta_{0}=0$ we have

$$
2 E_{0}=\phi_{\rho}^{2}+\phi_{0}^{2} .
$$

We now apply the maximum principle (Hopf's Lemma) to the uniformly elliptic equation (38), noting the boundary condition, and get

$$
\begin{gathered}
\phi_{0}=F_{0}(\theta), \\
E_{0}=0 .
\end{gathered}
$$

Let us proceed to the $\epsilon^{1}$ equations. In region $\mathrm{R}$

$$
\left(\frac{\partial}{\partial \rho} \rho \frac{\partial}{\partial \rho}+\frac{\partial}{\partial s} \rho \frac{\partial}{\partial s}\right) \phi_{1}=-\frac{\ddot{F}_{0}}{\rho} .
$$

On $s-G=0$

$$
\partial \phi_{1} / \partial n=0
$$

On $s-\eta_{0}=0$

$$
\phi_{1,}-\phi_{1_{\rho}} \eta_{0,}=\eta_{0 .}\left(\frac{\dot{F}_{0}}{\rho^{2}}-\bar{\omega}\right)
$$

and

$$
\eta_{0}=E_{1}+\dot{F}_{0}\left(\bar{\omega}-\frac{\dot{F}_{0}}{2 \rho^{2}}\right) .
$$


Eqs. (45) and (46) combine to give us Eq. (47) on $s=\eta_{0}$ :

$$
\phi_{1},-\phi_{1}, \eta_{0_{\rho}}=-\ddot{F}_{0}\left(\frac{\dot{F}_{0}}{2}-\tilde{\omega}\right)^{2} \text {. }
$$

We apply the divergence theorem on Eqs. (43), (44) and (47) and get

$$
\begin{aligned}
F_{0} & =\gamma \theta, \\
\phi_{1} & =F_{1}(\theta), \\
\eta_{0} & =E_{1}+\gamma\left(\bar{\omega}-\frac{\gamma}{2 \rho^{2}}\right) .
\end{aligned}
$$

At this point it is clear which region we are dealing with (see Fig. 2). Here $a, a+b$ denote the radial extent of the steady-flow fluid and $b$ is the width of the channel. The admissibility of vertical-sided channels of width $b$ is also evident. We see that $\left\{\hat{\phi} \equiv \phi_{0}\right.$; $\left.\hat{\eta} \equiv \eta_{0}\right\}$ is an exact solution of this fluid flow problem and is the steady-flow state previously defined. From the equations coefficient to $\epsilon^{2}$ we get

$$
\left(\frac{\partial}{\partial \rho} \rho \frac{\partial}{\partial \rho}+\frac{\partial}{\partial s} \rho \frac{\partial}{\partial s}\right) \phi_{2}=-\frac{\ddot{F}_{1}}{\rho}
$$

in R. On $s=G$ we get

$$
\partial \phi_{1} / \partial n=0 .
$$

On $s=\eta_{0}$ we get Eqs. (53) and (54) which combine to (55):

$$
\phi_{2,}-\phi_{2,} \eta_{0_{\rho}}=\eta_{1},\left(\frac{\gamma}{\rho^{2}}-\bar{\omega}\right),
$$

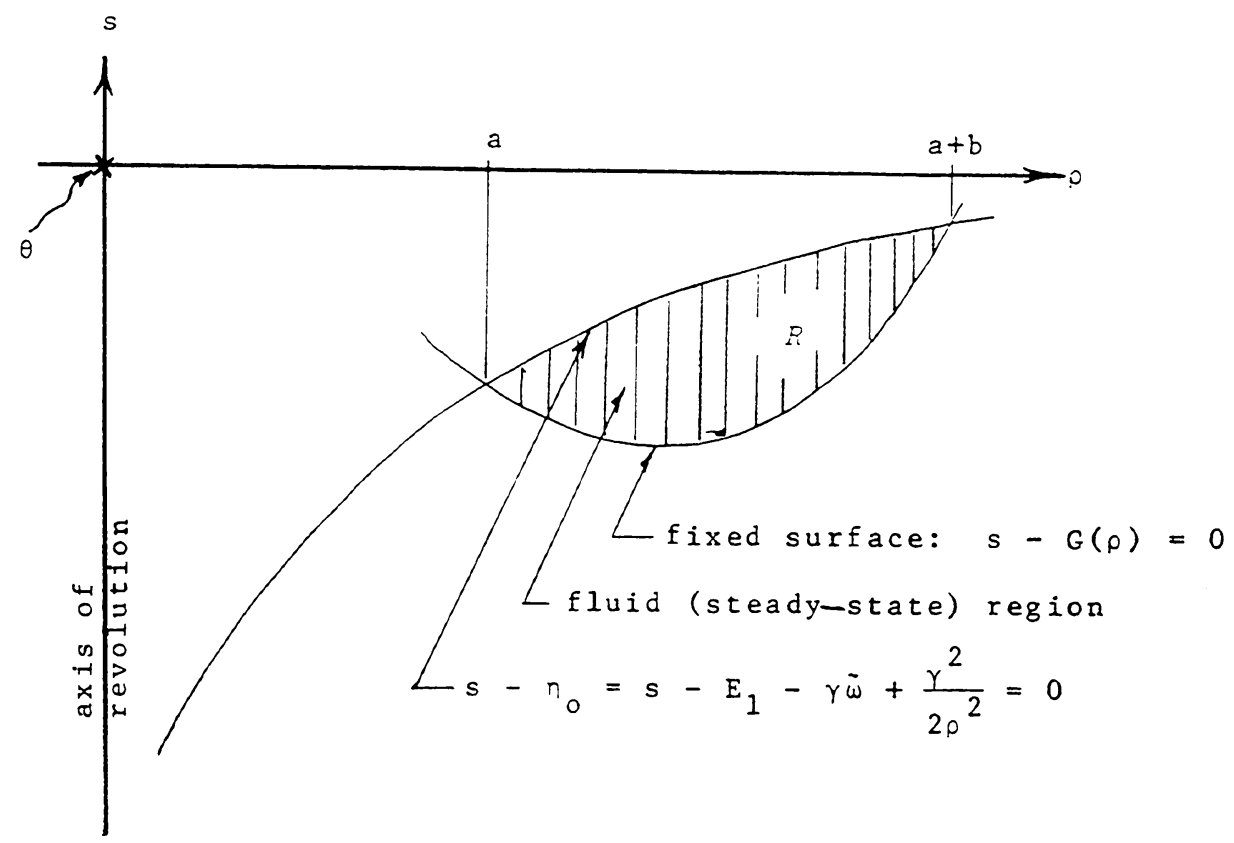

Fig. 2. Region $R$. 


$$
\begin{gathered}
\eta_{1}=E_{2}+\dot{F}_{1}\left(\bar{\omega}-\frac{\gamma}{\rho^{2}}\right) \\
\phi_{2,}-\phi_{2 \rho} \eta_{0_{\rho}}=-\ddot{F}_{1}\left(\bar{\omega}-\frac{\gamma}{\rho^{2}}\right)^{2} .
\end{gathered}
$$

Applying the divergence theorem to Eqs. (51), (52) and (55), we get

$$
\ddot{F}_{1}\left(\iint_{R}(1 / \rho) d A-\int_{a}^{a+b} \rho\left(\bar{\omega}-\frac{\gamma}{\rho^{2}}\right)^{2} d \rho\right)=0 .
$$

Eq. (56) represents a bifurcation typical of this problem which also appears in the case of rotational waves and in the case of waves of variable-density fluid.

If $\left(\ddot{F}_{1} \equiv 0\right)$, we get irrotational flow with no wave, i.e. the trivial steady-flow solution. That is,

$$
\begin{aligned}
\eta & =\eta_{0}+\epsilon \eta_{1}+O\left(\epsilon^{2}\right) \\
& =\left(E_{1}+\gamma\left(\bar{\omega}-\frac{\gamma}{2 \rho^{2}}\right)\right)+\epsilon\left(E_{2}+\gamma_{1}\left(\tilde{\omega}-\frac{\gamma}{\rho^{2}}\right)\right)+O\left(\epsilon^{2}\right) \\
& =\eta(\rho)+O\left(\epsilon^{2}\right)
\end{aligned}
$$

where $\dot{F}_{1} \equiv \gamma_{1}=$ constant. If we make similar assumptions throughout the expansion, we get $\eta_{i}=\eta_{i}(\rho)$. The final result would then be $\eta=\eta(\rho ; \epsilon)=A+B \rho^{-2}$ where both $A$ and $B$ are arbitrary constants with respect to the space and time variables.

This is a steady-state solution and satisfies the exact differential equations of motion. In fact, it can be represented as $\eta=\eta_{0}(\rho)$. For the solutions which may be waves we are forced to look at the alternate solutions of Eq. ( 56$)$. Assume $\left(\ddot{F}_{1} \not \equiv 0\right)$; then $\gamma$ is determined from

$$
\left(\iint_{\mathrm{R}}(1 / \rho) d A-\int_{0}^{a+b} \rho\left(\tilde{\omega}-\frac{\gamma}{\rho^{2}}\right)^{2} d \rho\right)=0
$$

or by integrating once we get $\gamma(\tilde{\omega})$ defined implicitly by

$$
\int_{a}^{a+b}\left(\frac{G-E_{1}}{\rho}+\frac{3 \gamma^{2}}{2 \rho^{3}}-\frac{3 \gamma \tilde{\omega}}{\rho}+\rho \bar{\omega}^{2}\right) d \rho=0 .
$$

Integrating again we get a second-order equation in $\gamma$ and $\tilde{\omega}$ :

$\tilde{\omega}^{2}-(\tilde{\omega} \gamma)\left[\frac{3 \ln (1+b / a)}{a b(1+b / 2 a)}\right]+\frac{3 \gamma^{2}}{2 a^{4}(1+b / a)^{2}}=\frac{1}{a b(1+b / 2 a)} \int_{a}^{a+b}\left(E_{1}-G\right) / \rho d \rho$.

Two pairs of solutions are of special interest. The first is the case of stationary waves. If $\bar{\omega}=0$, then

$$
\gamma= \pm\left(\frac{2 a^{3}(1+b / a)^{2}}{3 b(1+b / 2 a)} \int_{a}^{a+b}\left(E_{1}-G\right) / \rho d \rho\right)^{1 / 2}
$$

i.e., there are two associated steady-flows, one clockwise and one counterclockwise. Both are the same in absolute value. Another interesting solution is a perturbation from a state where there is no steady-flow motion. Suppose $\gamma=0$; then the bulk of the fluid has no motion (w.r.t. $O(\epsilon)$ ) and 


$$
\bar{\omega}= \pm\left(\frac{1}{a b(1+b / 2 a)} \int_{a}^{a+b}\left(E_{1}-G\right) / \rho d \rho\right)^{1 / 2} .
$$

In this case we get a clockwise and a counterclockwise wave with the same wave velocity amplitude.

It is interesting to look at the solutions as $a$ gets very large with $b$ relatively fixed and keeping the same channel contour $G$. Let HT be the average height of fluid in such a channel. As a result, Eq. (58) gives us the relation

$$
(a \tilde{\omega}-\gamma / a)^{2} \rightarrow(\mathrm{HT}) \quad \text { as } \quad(a) \rightarrow \infty .
$$

In dimensional form this statement says that the square of the relative velocity of the wave to the bulk of fluid approaches (w.r.t. $O(\epsilon)$ ) the product of the average height $\mathrm{HT}$ and the acceleration of gravity $g$. This is the same as the critical-speed expression for infinite straight channels. We adopt the terms critical speed or critical velocity to the case of circular channels, since it is an obvious extension of the term defined for twodimensional waves.

Another point that should be made concerning Eq. (58) or (60) and Fig. 2 is that, in general, one cannot pick $a$ and $b$ arbitrarily. One can easily see this by noting that the following inequality must hold:

$$
G(a+b)=\eta_{0}(a+b) \geq \eta_{0}(a)=G(a) .
$$

Let us pick a set $(a, b)$ such that inequality $(64)$ holds. Then from Eq. (50) there will be one solution only for $\gamma$ and $E_{1}+\gamma \tilde{\omega}$. With $\gamma$ thus fixed we now solve Eq. (60) for two values of $\tilde{\omega}$. One wave will be going faster than the bulk of fluid, and one wave will be going slower and perhaps in the opposite direction. For each of these values of $\bar{\omega}$ we have a corresponding $E_{1}$, and hence all the low-order parameters that we need are fixed. There is the possibility of $G(\rho)=\eta_{0}(\rho)$ for some $\rho$ given by $a<\rho<a+b$. In such a case the region $\mathrm{R}$ will be decomposed into two or more disjoint regions, and each region should be treated as a separate channel. Keeping the above in mind, we return to Eqs. (51), (54) and (55) and seek a solution under the condition that $\ddot{F}_{1} \not \equiv 0$ or Eq. (58). Using the maximum principle, we see that $\phi_{2}$ may be written as

$$
\phi_{2}(\rho, \theta, s)=\ddot{F}_{1}(\theta) \Psi(\rho, s)+F_{2}(\theta)
$$

where $F_{2}(\theta)$ is as yet undetermined and $\Psi(\rho, s)$ is a solution of Eqs. (66), (67) and (68). In region $R$

$$
\left(\frac{\partial}{\partial \rho} \rho \frac{\partial}{\partial \rho}+\frac{\partial}{\partial s} \rho \frac{\partial}{\partial s}\right) \Psi=-\frac{1}{\rho} .
$$

On $s=G$,

$$
\partial \Psi / \partial n=0 .
$$

On $s=\eta_{0}$,

$$
\Psi_{\triangleleft}-\Psi_{\rho} \eta_{0_{\rho}}=-\left(\tilde{\omega}-\frac{\gamma}{\rho_{2}}\right)^{2} .
$$

In order to get more information about $F_{1}(\theta)$ we go to the next higher-order equations, i.e., those coefficient to $\epsilon^{3}$. On $R$ we have

$$
\left(\frac{\partial}{\partial \rho} \rho \frac{\partial}{\partial \rho}+\frac{\partial}{\partial s} \rho \frac{\partial}{\partial s}\right) \phi_{3}=-\frac{\phi_{2 \theta \theta}}{\rho}=-\frac{\dddot{F}_{1} \Psi}{\rho}-\frac{\ddot{F}_{2}}{\rho} .
$$


On $s=G$,

$$
\frac{\partial \phi_{3}}{\partial n}=0
$$

We have

$$
\eta_{2}=E_{3}-\frac{\dot{F}_{1}^{2}}{2 \rho^{2}}+\left(\tilde{\omega}-\frac{\gamma}{\rho^{2}}\right)\left(\dot{F}_{2}+\ddot{F}_{1} \Psi\left(\rho, \eta_{0}\right)\right)
$$

and on $s=\eta_{0}$ we get

$$
\begin{aligned}
\left(\phi_{3,}-\phi_{3_{\rho}} \eta_{\rho_{\rho}}\right)= & \dddot{F}_{1} \cdot\left(-\Psi\left(\tilde{\omega}-\frac{\gamma}{\rho^{2}}\right)^{2}\right)+\ddot{F}_{1} E_{2}\left(\frac{\Psi_{\rho s} \gamma^{2}}{\rho^{3}}-\Psi_{s s}\right), \\
& +\dot{F}_{1} \ddot{F}_{1}\left[\left(\tilde{\omega}-\frac{\gamma}{\rho^{2}}\right)\left(\frac{2}{\rho^{2}}-\Psi_{s s}+\frac{\gamma^{2} \Psi_{\rho s}}{\rho^{3}}\right)+\frac{2 \gamma \Psi_{\rho}}{\rho^{3}}\right]-\ddot{F}_{2}\left(\tilde{\omega}-\frac{\gamma}{\rho^{2}}\right)^{2} .
\end{aligned}
$$

Applying the divergence theorem to the above we get a differential equation for $F_{1}$ :

$$
\begin{aligned}
\dddot{F}_{1} \cdot\left[\iint_{\mathrm{R}} \frac{\Psi}{\rho} d A-\left.\int_{a}^{a+b} \Psi\right|_{s=\eta_{0}}\left(\bar{\omega}-\frac{\gamma}{\rho^{2}}\right)^{2} \rho d \rho\right] \\
=\ddot{F}_{2}\left[\int_{a}^{a+b}\left(\bar{\omega}-\frac{\gamma}{\rho^{2}}\right)^{2} \rho d \rho-\iint \frac{d A}{\rho}\right]+\left.E_{2} \ddot{F}_{1} \int_{a}^{a+b} \rho\left[\Psi_{s s}-\frac{\Psi_{\rho s} \gamma^{2}}{\rho^{3}}\right]\right|_{s=\eta_{0}} d \rho \\
\quad+\left.\dot{F}_{1} \ddot{F}_{1} \int_{a}^{a+b} \rho\left[\left(\bar{\omega}-\frac{\gamma}{\rho^{2}}\right)\left(\Psi_{s s}-\frac{2}{\rho^{2}}-\frac{\gamma^{2} \Psi_{\rho s}}{\rho^{3}}\right)-\frac{2 \gamma \Psi_{\rho}}{\rho^{3}}\right]\right|_{s=\eta_{0}} d \rho .
\end{aligned}
$$

The coefficient of $F_{2}$ in Eq. (73) is zero because of Eq. (58). We can further simplify the above using the following definitions for $m_{0}, m_{1}, m_{2}$. The result, after integrating the leading term, is Eq. (75). Let

$$
\begin{aligned}
& m_{0} \equiv \iint_{\mathrm{R}} \rho\left[\Psi_{s}^{2}+\Psi_{\rho}^{2}\right] d A>0 \\
& \left.m_{1} \equiv \int_{a}^{a+b}\left[\left(\tilde{\omega}-\frac{\gamma}{\rho^{2}}\right)\left(\rho \Psi_{s s}-\frac{2}{\rho}-\frac{\gamma^{2} \Psi_{\rho s}}{\rho^{2}}\right)-\frac{2 \gamma \Psi_{\rho}}{\rho^{2}}\right]\right|_{.=\eta_{0}} d \rho, \\
& \left.m_{2} \equiv E_{2} \int_{a}^{a+b}\left(\rho \Psi_{a}-\frac{\Psi_{\rho s} \gamma^{2}}{\rho^{2}}\right)\right|_{s=\eta_{0}} d \rho .
\end{aligned}
$$

Then

$$
m_{0} \ddot{F}_{1}=m_{1} \dot{F}_{1} \ddot{F}_{1}+m_{2} \ddot{F}_{1} \text {. }
$$

We can solve for $F_{1}$ exactly with Eq. (75) once we know $m_{0}, m_{1}, m_{2}$ explicitly. These values depend upon $\Psi(\rho, s)$, which is a solution of Eqs. (66), (67) and (68), and hence depend upon the particular channel shape $s=G(\rho)$. Rather than getting explicit solutions for various channels we can integrate (75) directly and get solutions for $F_{1}$ in terms of the coefficients $m_{0}, m_{1}, m_{2}$. Since the general properties of $F_{1}$ are easily determined, the wave shape will thus be established. We will then get expressions for the velocities $V_{1}, V_{2}, V_{3}$.

Wave shapes. The solutions of (75) fall into three categories depending on the coefficient of the nonlinear term $m_{1}$. Of these, the third case is the most interesting. 
Case 1. Let $m_{1}=0$. Eq. (75) is now linear and has smooth solution only if $m_{2}<0$ :

$$
\dot{F}_{1}=\left(\left(r_{1}+r_{2}\right) / 2\right)+\left(\left(r_{2}-r_{1}\right) / 2\right) \cos \left(\left(-m_{2} / m_{0}\right)^{1 / 2} \theta\right) \text {. }
$$

There must be a further restriction on $m_{2} / m_{0}$ so that $\eta_{1}$ will be continuous. This is given by $(77 a)$ or $(77 b)$ :

$$
\begin{aligned}
& -j^{2} m_{0}=m_{2} \epsilon ; \quad j=1.2,3, \cdots, \\
& -j^{2}\left[\iint_{\mathrm{R}} \rho\left[\Psi_{s}^{2}+\Psi_{\rho}^{2}\right] d A\right]=\left.\epsilon E_{2} \int_{a}^{n+b}\left(\rho \Psi_{s}-\frac{\gamma^{2} \Psi_{\rho s}}{\rho^{2}}\right)\right|_{s-n_{0}} d \rho<0 .
\end{aligned}
$$

Under these restrictions we get

$$
\dot{F}_{1}=\left(\left(r_{1}+r_{2}\right) / 2\right)+\left(\left(r_{2}-r_{1}\right) / 2\right) \cos j \alpha,
$$

or

$$
\begin{aligned}
\eta= & \eta_{0}+\epsilon \eta_{1}+O\left(\epsilon^{2}\right) \\
= & E_{1}+\gamma\left(\frac{\tilde{\Omega}}{\sqrt{ } g}-\frac{\gamma}{2 \rho^{2}}\right)-j^{2}\left[\frac{\iint_{R}\left[\Psi_{s}^{2}+\Psi_{\rho}^{2}\right] \rho d A}{\left.\int_{a}^{a+b}\left(\rho \Psi_{s s}-\frac{\gamma^{2} \Psi_{\rho s}}{\rho^{2}}\right)\right|_{s-\eta_{0}} d \rho}\right] \\
& \cdot\left[1+\left(\frac{\tilde{\Omega}}{\sqrt{ } g}-\frac{\gamma}{\rho^{2}}\right)\left(\frac{\hat{r}_{1}+\hat{r}_{2}}{2}\right)+\left(\frac{\hat{r}_{2}-\hat{r}_{1}}{2} \cos j(\alpha-\tilde{\Omega} t)\right)\right]+O\left(\epsilon^{2}\right)
\end{aligned}
$$

where $\hat{r}_{1}$ and $\hat{r}_{2}$ are arbitrary constants. Therefore we may specify the number of waves in the channel or bowl by specifying $j$ and we may specify the wave height in Eq. (79) by specifying $\hat{r}_{1}$ and $\hat{r}_{2}$. We note again that this solution is valid only for $m_{1}=0$ and $m_{2}<0$. The corresponding velocities will be given by

$$
\begin{aligned}
V_{1}= & j^{3} \sqrt{ } g\left[\frac{\hat{r}_{2}-\hat{r}_{1}}{2}\right]\left[\frac{\iint_{\mathrm{R}}\left[\Psi_{s}^{2}+\Psi_{\rho}^{2}\right] \rho d A}{\left.\int_{a}^{a+b}\left(\rho \Psi_{s s}-\frac{\gamma^{2} \Psi_{\rho s}}{\rho^{2}}\right)\right|_{s=\eta_{o}} d \rho}\right] \Psi_{\rho} \sin j(\alpha-\tilde{\Omega} t)+O\left(\epsilon^{5 / 2}\right), \\
V_{2}= & \frac{\sqrt{ } g}{\rho}\left[\gamma-\left\{\frac{j^{2} \iint_{\mathrm{R}}^{\rho} \rho\left[\Psi_{s}^{2}+\Psi_{\rho}^{2}\right] d A}{\left.\int_{a}^{a+b}\left(\rho \Psi_{s s}-\frac{\gamma^{2} \Psi_{\rho s}}{\rho^{2}}\right)\right|_{s=\eta_{o}} d \rho}\right\}\right. \\
& \left.\cdot\left\{\left(\frac{\hat{r}_{1}+\hat{r}_{2}}{2}\right)+\left(\frac{\hat{r}_{2}-\hat{r}_{1}}{2}\right)\left(1+j^{2} \Psi\right) \cos j(\alpha-\tilde{\Omega} t)+\epsilon^{2} F_{2}\right\}\right]+O\left(\epsilon^{3}\right),
\end{aligned}
$$

and

$$
V_{3}=j^{3} \sqrt{ } g\left[\frac{\hat{r}_{2}-\hat{r}_{1}}{2}\right]\left[\frac{\iint_{\mathrm{R}}\left[\Psi_{s}^{2}+\Psi_{\rho}^{2}\right] \rho d A}{\left.\int_{a}^{a+b}\left(\rho \Psi_{s s}-\frac{\gamma \Psi_{\rho s}}{\rho^{2}}\right)\right|_{s=\eta_{0}} d \rho}\right] \Psi_{s} \sin j(\alpha-\tilde{\Omega} t)+O\left(\epsilon^{5 / 2}\right) .
$$


Case 2. Let $m_{1}>0$. Let $\hat{F}_{1}=-F_{1}$ and substitute into Eq. (75). The differential equation for $\hat{F}_{1}$ will now fall into the category of Case 3.

Case 3. Let $\hat{M}_{1}<0$. To get the simplest form for the solution we let

$$
Z=\dot{F}_{1}+m_{2} / m_{1} ;
$$

then

$$
m_{0} \ddot{Z}^{\cdot}=m_{1} Z \dot{Z}
$$

Let

$$
\lambda \equiv-3 m_{0} / m_{1}=\frac{3 \iint_{\mathrm{R}} \rho\left[\Psi_{s}^{2}+\Psi_{\rho}^{2}\right] d A}{\left.\int_{a}^{a+b}\left[\left(\tilde{\omega}-\frac{\gamma}{\rho}\right)\left(\frac{2}{\rho}+\frac{\gamma^{2} \Psi_{\rho s}}{\rho^{2}}-\rho \Psi_{\iota s}\right)+\frac{2 \gamma \Psi \rho}{\rho^{2}}\right]\right|_{l,-\eta_{0}} d \rho}>0
$$

and

$$
\hat{C} \equiv-\frac{m_{2}}{\gamma E_{2} m_{1}}=\frac{\left.\int_{a}^{a+b}\left(\rho \Psi_{s s}-\frac{\gamma^{2} \Psi_{\rho s}}{\rho^{2}}\right)\right|_{s-\eta_{0}} d \rho}{\left.\gamma \int_{a}^{a+b}\left[\left(\bar{\omega}-\frac{\gamma}{\rho^{2}}\right)\left(\frac{2}{\rho}+\frac{\gamma^{2} \Psi_{\rho s}}{\rho^{2}}-\rho \Psi_{s s}\right)+\frac{2 \gamma \Psi \rho}{\rho^{2}}\right]\right|_{s-\eta_{0}} d \rho} .
$$

The equation for the free surface then becomes

$$
\eta=\eta_{0}+\epsilon E_{2}\left[1+\gamma \hat{C}\left(\bar{\omega}-\frac{\gamma}{\rho^{2}}\right)\right]+\epsilon Z\left(\bar{\omega}-\frac{\gamma}{\rho^{2}}\right)+O\left(\epsilon^{2}\right) .
$$

Let

$$
\begin{aligned}
j & =\text { number of wave crests }=1,2,3, \cdots, \\
H & =\text { height factor, } \\
r & =\text { depth/height ratio, } \\
k^{2} & =(1+r) /(2-r), \\
\xi & =2-r .
\end{aligned}
$$

Then Eq. (84) may be integrated provided the value of $r$ and hence the values of $\xi$ and $k^{2}$ are restricted as in Table I.

\section{TABLE I}

$$
\begin{array}{l|l|}
\hline r=0 & 0<r<\frac{1}{2} \\
\hline k^{2}=\frac{1}{2} & \begin{array}{l}
2<k^{2}<1 \\
\hline \xi=2
\end{array} \\
\hline 2>\xi>\frac{3}{2} \\
\hline
\end{array}
$$

Let $\Lambda$ be defined as

$$
\Lambda \equiv a m\left(\theta\left(\frac{H \xi}{4 \lambda \epsilon}\right)^{1 / 2}, k\right) \equiv a m\left(\alpha\left(\frac{H \xi}{4 \lambda}\right)^{1 / 2}, k\right)
$$


where

$$
\alpha\left(\frac{H \xi}{4 \lambda}\right)^{1 / 2} \equiv \theta\left(\frac{H \xi}{4 \lambda \epsilon}\right)^{1 / 2}=\int_{0}^{\Lambda} \frac{d \Lambda}{\left(1-k^{2} \sin ^{2} \Lambda\right)^{1 / 2}}
$$

then

$$
\begin{aligned}
& c n(A, B) \equiv \cos \Lambda \equiv \cos (a m(A, B)), \\
& \operatorname{sn}(A, B) \equiv \sin \Lambda \equiv \sin (a m(A, B)) .
\end{aligned}
$$

With these definitions Eq. (84) integrates to

$$
Z=\frac{H}{\epsilon}\left[-r+(1+r) n^{2}\left(\alpha\left(\frac{H \xi}{4 \lambda}\right)^{1 / 2}, k\right)\right] .
$$

From continuity conditions for the wave, it is seen that $j, H$, and $k$ are connected through

$$
\left(\frac{\pi}{2 j}\right)\left(\frac{3 H}{\lambda\left(k^{2}+1\right)}\right)^{1 / 2}=K(k)
$$

where $K(k)$ is the complete elliptic integral of the first kind. We may arbitrarily choose $j$, the number of waves, and one other parameter from the following group $H, k, r, \xi$. Eqs. (88) and (93) imply that the rest of the parameters are determined provided they are confined to the range in Table I.

The equation for the free surface then becomes

$$
\begin{aligned}
\eta= & E_{1}+\gamma\left(\frac{\tilde{\Omega}}{\sqrt{ } g}-\frac{\gamma}{2 \rho^{2}}\right)+\epsilon E_{2}\left[1+\gamma \hat{C}\left(\frac{\tilde{\Omega}}{\sqrt{ } g}-\frac{\gamma}{2 \rho^{2}}\right)\right] \\
& +\left(\frac{\tilde{\Omega}}{\sqrt{ } g}-\frac{\gamma}{\rho^{2}}\right) H\left((1+r) \operatorname{cn}^{2}\left(\frac{\alpha j K(k)}{\pi}, k\right)-r\right)+O\left(\epsilon^{2}\right) .
\end{aligned}
$$

Notice that $E_{2}$ is still arbitrary. Various criteria may be used to fix its value. When considering higher-order terms it is best to set it equal to zero. The nature of the wave is independent of its value and is shown in Eq. (95):

$$
\operatorname{SHAPE}(\eta)=H\left(\frac{\tilde{\Omega}}{\sqrt{ } g}-\frac{\gamma}{\rho^{2}}\right)\left[(1+r) \operatorname{cn}^{2}\left(\frac{\alpha j K(k)}{\pi}, k\right)-r\right] \text {. }
$$

The definitions for $H, r, j$ in relations (88) are now apparent.

Eqs. (96), (97) and (98) represent the three components of velocity for this case. Eqs. (90) and (85) may be used as a reference for the values of $\Lambda(\alpha)$ and $\lambda$.

$$
\begin{aligned}
V_{1}= & -\left(\frac{H^{3} g(2-r)}{4 \lambda}\right)^{1 / 2}(1+r) \sin 2 \Lambda\left(1-k^{2} \sin ^{2} \Lambda\right)^{1 / 2} \Psi_{\rho}+O(\epsilon)^{5 / 2}, \\
V_{2}= & \frac{\sqrt{ } g}{\rho}\left[\gamma+H(1+r) \cos ^{2} \Lambda-r\right)+\frac{H^{2}}{2 \lambda}(1+r)(2-r) \\
& \left.\cdot\left\{\frac{k^{2} \sin ^{2} 2 \Lambda}{4}-\cos 2 \Lambda\left(1-k^{2} \sin ^{2} \Lambda\right)\right\}+\epsilon E_{2} \gamma \hat{C}+\epsilon^{2} \dot{F}_{2}\right]+O\left(\epsilon^{3}\right), \\
V_{3}= & -\left(\frac{H^{3} g(2-r)}{4 \lambda}\right)^{1 / 2}(1+r) \sin 2 \Lambda\left(1-k^{2} \sin ^{2} \Lambda\right)^{1 / 2} \Psi_{\iota}+O(\epsilon)^{5 / 2},
\end{aligned}
$$




$$
(\alpha-\tilde{\Omega} t)\left(\frac{H(2-r)}{4 \lambda}\right)^{1 / 2}=\int_{0}^{\Lambda} \frac{d \Lambda}{\left(1-k^{2} \sin ^{2} \Lambda\right)^{1 / 2}} .
$$

Higher-order terms may likewise be determined.

Rotational waves. Rotational waves may be treated in very similar ways. Eqs. (1) through (21) remain the same. The variables $U, V, W, \eta, \pi$ are expanded in formal power series in $\epsilon$. The techniques used in solving the resulting differential equations vary with the type of steady-flow state. In all the cases I have solved, however, three familiar results appear: first, the bifurcation; second, the critical speed condition; and last, the cnoidal wave shape. In most cases there is a horrendous bookkeeping problem with lengthy equations. The irrotational case, is, however, representative as far as wave shape is concerned.

Limiting case: straight channel. In the first section of this paper we noted the lack of an existence theorem for the progressing waves described here. In order to establish a good degree of credence to this method we can easily show that these solutions transform smoothly into the solutions for straight channels as we straighten out the circular channels referred to in this paper. Since there is an existence theorem [3], [4] for the solution in the straight channel, we might expect existence for the waves discussed in this report. We proceed by fixing both the value $b$ and the cross-section shape $G$, and then we let $a$ approach infinity. We then compare our solutions to the solutions discussed by Peters [2] in straight channels. After suitably identifying terms, we see that the coefficients $m_{0}, m_{1}, m_{2}$ and others smoothly transform into their proper limits. Since the wave shape is a smooth function of these parameters, we see that it also transforms into the expected limit. As a result, we see that as the channel straightens out ( $b, G$ fixed: $a \rightarrow \infty$ ), the cnoidal waves discussed here transform into the cnoidal waves for infinite straight channels.

\section{BibLIOGRAPHY}

[1] J. J. Stoker, Jr., Water waves: the mathematical theory with applications, Pure and Appl. Math., vol. 4, Interscience, New York, 1957

[2] A. S. Peters, Rotational and irrotational solitary waves in a channel with arbitrary cross section, Comm. Pure Appl. Math. 19, 445-471 (1966)

[3] K. O. Friedrichs and D. H. Hyers, The existence of solitary waves, Comm. Pure Appl. Math. 7, 517550 (1954)

[4] W. Littman, On the existence of periodic waves near critical speed, Comm. Pure Appl. Math. 10, 241269 (1957) 